\title{
Editorial
}

\section{Cognitive Therapy and Interpersonal Psychotherapy: 30 Years Later}

I.

t has been a good year for psychotherapy. Aaron Beck won the prestigious Albert Lasker Award for Clinical Medical Research for the development of cognitive therapy, giving psychotherapy new recognition in the scientific community. In his acceptance speech, Beck noted that if his young postdoc John Rush hadn't prodded him, cognitive therapy would not have gone anywhere. Rush told him that no one would believe his new therapy was effective until he did a clinical trial.

Around the same time, Gerry Klerman was planning the first large maintenance medication trial of depression. Most patients receiving medication also received psychotherapy, and Klerman wanted to mimic clinical practice. He admired Beck's manual, but most patients, he said, were receiving supportive therapy, which was undefined and untested. He gave the Beck manual to his researchers and said, "Do something as specified as this for supportive psychotherapy." In the first draft of the manual, which we used in the maintenance trial, we called the treatment "high-contact psychotherapy." Later, after efficacy was demonstrated, we termed it interpersonal psychotherapy (IPT). Thirty years later, cognitive therapy and IPT re-

\begin{tabular}{c}
\hline "Thirty years after \\
cognitive therapy and IPT \\
were conceived, we are \\
still looking at dose, \\
effectiveness, and new \\
indications."
\end{tabular}
main the most widely tested psychotherapies for the treatment of unipolar major depression. In this issue, articles by Thase et al., Wisniewski et al., Frank et al., and Schramm et al. present refinement data on cognitive therapy and IPT in relation to medication-Thase and Wisniewski on effectiveness, Frank on dose, and Schramm on a new indication.

The Thase and Wisniewski papers derive from the Sequenced Treatment Alternatives to Relieve Depression trial (STAR*D), which was designed to determine what options should be considered in successive next steps for depressed outpatients who do not benefit adequately from an initial course of an antidepressant (1).

The Thase et al. article reports on the effectiveness of cognitive therapy as a secondstep strategy. STAR* $D$ 's design includes patients' choices of treatment within a randomized design for patients who did not remit on citalopram. Seven treatments were grouped into four treatment strategies: medication switch or augmentation and cognitive therapy switch or augmentation. Patients could elect not to be assigned to certain treatment strategies and were eligible to remain in the study as long as random assignment was possible for at least one strategy. For example, a patient could exclude all but medication switch options. Then the patient would be randomly assigned to one of the three medication treatments within the medication switch strategy. Another patient might exclude switching to cognitive therapy and switching medications. This patient would then be randomly assigned to one of three treatments from the two remaining treatment strategies-augmentation with cognitive therapy or medication. However, if a patient selected only a switch to cognitive therapy, the patient was excluded from the study because random assignment could not be achieved.

Cognitive therapy augmentation was found to be as effective as medication augmentation, but medication augmentation produced more rapid remission. Cognitive therapy switch and medication switch were equally effective, but a medication switch produced more side effects. For patients who did not achieve complete remission af-

This article is featured in this month's AJP Audio. 
ter 12-14 weeks on citalopram (about $60 \%$ ), medication and cognitive therapy were a draw. There were also few differences in patient outcome among the different medications $(1,2)$.

The surprising point in this article is not the outcome but the fact that only about a quarter of patients (26\%) chose cognitive therapy, either as an augmentation or a switching strategy. This low level of patient preference for cognitive therapy is at variance with earlier research by the same investigators. However, given design issues that may have biased the choice, all noted by the authors, we cannot conclude that most depressed patients do not want cognitive therapy. Patients who entered the study had to be willing to take medication as the only initial treatment option. Patients who were already participating in psychotherapy could be enrolled in $\operatorname{STAR}^{*} \mathrm{D}$, but when the time came for augmentation or switching, those who wished to continue their current psychotherapy may have declined random assignment to cognitive therapy because that would have meant giving up their relationship with an outside psychotherapist. There were also convenience issues. Patients receiving cognitive therapy had to go to an offsite office. The study did not reimburse for copayment charges for psychotherapy covered by insurance, whereas all medication charges were covered. Patients had already formed a relationship with their medication treating physician. If they chose augmentation, they could maintain that relationship. If they switched to cognitive therapy, that relationship was broken.

The Wisniewski et al. paper follows up the Thase et al. findings by seeking to understand patients' willingness to accept the various switching or augmenting second-step strategies. The factors related to the choice of cognitive therapy as a second-step treatment were better education and a family history of depression. Since education is associated with income, it is possible that this finding is a reflection of the need to pay for cognitive therapy not covered by insurance. Higher-income patients may have had better insurance coverage and might not have incurred much or any out-of-pocket expense. The relationship with family history is harder to interpret.

Patients' choice of a switch from the initial medication treatment made sense. Patients who had more side effects with, or obtained inadequate benefit from, the first medication wanted to switch to something different, usually a different medication. But patients did make choices. Few patients accepted all four strategies offered.

The article by Frank et al. addresses the "dose" of IPT. The study follows up their 3year maintenance trial of imipramine and IPT for ambulatory patients with severe recurrent major depression (3). Their original study had the highest dose of maintenance medication (imipramine at $200 \mathrm{mg}$ ) and the lowest dose of IPT (monthly) ever used for study patients with recurrent depression. The question they asked in the new study was whether increasing the frequency of maintenance IPT sessions would increase the therapy's prophylactic effect. Acutely depressed women who wanted to maintain remission without medication received weekly IPT alone in acute treatment. If they required medication for remission of acute symptoms ( $39 \%$ of the women did), they were given a selective serotonin reuptake inhibitor (SSRI) in addition to IPT. After remission the women were randomly assigned to IPT weekly, twice monthly, or monthly as their sole therapy for 2 years or until a recurrence. Overall, $26 \%$ of the women who received IPT only in the acute phase and nearly twice as many $(50 \%)$ of those who required the addition of an SSRI to IPT in the acute phase experienced a recurrence during maintenance treatment with IPT alone. However, in neither group did the frequency of IPT make a difference. For women who can achieve remission on IPT alone in acute treatment (and about two-thirds did), monthly IPT is a good maintenance treatment. But for those who need the addition of medication during acute treatment, IPT alone as maintenance treatment, regardless of IPT dose, is not a good choice.

The good news is that monthly IPT alone can maintain remission for 2 years in the majority of women who are able to achieve remission on it during the acute phase. As 
Ellen Frank has said, "What gets you well keeps you well." This is especially good news for many depressed women who want to become pregnant and nurse.

The Schramm et al. study, from Germany, tested a new indication and dose for IPTtreatment of inpatients with depression with a high dose of IPT. Hospitalized patients received either an intensive dose of individual and group IPT sessions along with pharmacotherapy or pharmacotherapy plus clinical management. IPT was delivered individually three times a week for 5 weeks, and eight group sessions were conducted during that period. Significant others were educated about the illness. The authors found that brief intensive IPT was superior to standard treatment for these severely ill hospitalized patients, and the differential effects lasted for 3 months. Schramm et al. conclude that it is best to begin intensive treatment early and not wait for a period of stabilization to add psychotherapy. This approach is quite feasible within the health insurance systems of Germany and some other countries, but less so in the United States, where few insurance plans will pay for this amount of hospitalization or even for this amount of psychotherapy for outpatients.

These four articles largely represent a shift of treatment goals from response to remission. Most depressed patients may need trials of different medications and/or psychotherapy to finally reach remission. Clinicians' adherence to a protocol, monitoring of the patient's symptoms during treatment, willingness to try different options, and sensitivity to the patient's preferences all help in achieving remissions that may at first be elusive.

Let us put the findings of these studies into the context of available treatments for depression. A choice of medications was usually included, and the differences in outcome between medications were small on direct comparison. However, patients' responses to a change in medication as an augmentation or a switch given in sequence were not. It would be incorrect to dismiss various antidepressants as all "me too."

There are clear differences between the two psychotherapies used in these studies. Cognitive therapy addresses distorted, negative thinking associated with depression, whereas IPT addresses stressful social and interpersonal relationships associated with the onset of depressive symptoms. The therapies have been differentiated by blind raters viewing tapes of actual sessions. Some differences in outcomes and predictors have been shown in the one large study in which direct comparisons were made (4). Some psychotherapy experts believe that all psychotherapies resemble each other and have a broad common element of good sense and intentions. But, as with medications, there may be important clinical differences between them.

Unlike medication, few psychotherapies for depression have undergone extensive testing in clinical trials. Psychotherapy has been a cottage industry, without any pharmaceutical company-like support for developing and testing new psychotherapies or new indications for old ones. Many excellent widely used psychotherapies remain untested, and tests directly comparing psychotherapies are rare. Thirty years after cognitive therapy and IPT were conceived, we are still looking at dose, effectiveness, and new indications. If these two psychotherapies were medications, by now there would be offlabel uses, generic versions, and numerous other second- and third-generation psychotherapies in trials.

As the study by Frank et al. shows, psychotherapy could have been offered as the first option in STAR*D. The argument that psychotherapy is not common community practice really depends on what community one looks at. If all the health professionals treating depression are included, a different conclusion may be drawn. A representative survey conducted in the United States in 1997 found that $74 \%$ of adults presenting for outpatient treatment of depression received an antidepressant and $60 \%$ received psychotherapy (5).

The next STAR*D-like clinical trial might have the option of beginning with psychotherapy alone or in combination with medication. However, this approach might face 
practical problems in clinical implementation. While psychiatry has taken a step forward with its new resident training requirements for teaching cognitive therapy, many in the psychotherapy workforce are not psychiatrists and do not receive information on medication options or training in evidence-based psychotherapies-even the Lasker award-winning cognitive therapy (6). The four articles in this issue support the value of new training efforts in multiple evidence-based psychotherapies.

\section{References}

1. Rush AJ, Trivedi MH, Wisniewski SR, Stewart JW, Nierenberg AA, Thase ME, Ritz L, Biggs MM, Warden D, Luther JF, Shores-Wilson K, Niederehe G, Fava M; STAR*D Study Team: Bupropion-SR, sertraline, or venlafaxine-XR after failure of SSRIs for depression. N EngI J Med 2006; 354:1231-1242

2. Trivedi MH, Fava M, Wisniewski SR, Thase ME, Quitkin F, Warden D, Ritz L, Nierenberg AA, Lebowitz BD, Biggs MM, Luther JF, Shores-Wilson K, Rush AJ; STAR*D Study Team: Medication augmentation after the failure of SSRIs for depression. N Engl I Med 2006; 354:1243-1252

3. Frank E, Kupfer DJ, Perel JM. Cornes C, Jarrett DB, Mallinger AG, Thase ME, McEachran AB, Grochocinski VJ: Three-year outcomes for maintenance therapies in recurrent depression. Arch Gen Psychiatry 1990; 47 : 1093-1099

4. Elkin 1, Gibbons RD, Shea MT, Sotsky SM, Watkins JT, Pilkonis PA, Hedeker D: Initial severity and differential treatment outcomes in the National Institute of Mental Health Treatment of Depression Collaborative Research Program. J Consult Clin Psychol 1995; 63:841-847

5. Olfson M, Marcus SC, Druss B, Elinson L, Tanielian T, Pincus HA: National trends in the outpatient treatment of depression. JAMA 2002; 287:203-209

6. Weissman MM, Verdeli H, Gameroff MJ, Bledsoe SE, Betts K, Mufson L, Fitterling H, Wickramaratne P: National survey of psychotherapy training in psychiatry, psychology, and social work. Arch Gen Psychiatry 2006 ; $63: 925-934$

MYRNA M. WEISSMAN, PH.D.

Address correspondence and reprint requests to Dr. Weissman, Chief, Division of Clinical and Genetic Epidemiology, New York State Psychiatric Institute, 1051 Riverside Dr., Unit 24, New York, NY 10032; mmw3@columbia.edu (e-mail).

Dr. Weissman has received investigator-initiated research support from NIMH, the Josiah J. Macy Foundation, NARSAD, GlaxoSmithKline, and Eli Lilly and is a developer of interpersonal psychotherapy. Dr. Freedman has reviewed this editorial and found no evidence of influence from these relationships. 